\title{
Acute Haemorrhagic Pancreatitis Leading to Sudden Unexpected Death - A Case Report and Review of Literature
}

Shetty $\mathrm{CK}^{1 *}$, Shetty $\mathrm{SS}^{2}$

$$
\text { Case Report }
$$

${ }^{1}$ Medical Lecturer, Department of Forensic Medicine, Faculty of Medicine, Universiti Sultan Zainal Abidin (Unisza), Kampus Kota, Kuala Terengganu, Malaysia.

${ }^{2}$ Medical Lecturer, Department of Surgery, Faculty of Medicine, UCSI University, Kampus Kuala Terengganu, Terengganu, Malaysia.

\begin{abstract}
Sudden death in young individuals is relatively uncommon and acute haemorrhagic pancreatitis as a cause of sudden and unexpected death is a rare phenomenon. The possible causal link between damage to the heart and acute pancreatitis and other pancreatic diseases has been considered in both adults and children, particularly in cases of sudden, unexpected death. However, the cardiac pathological findings so far reported in the literature are neither specific enough, nor of a kind to prove a direct pancreatic pathogenesis. A case of sudden death due to fatal myocardial infarction secondary to acute haemorrhagic pancreatitis is reported in a young male who was found dead at his house. The condition was discovered only at autopsy.
\end{abstract}

Keywords: Sudden Death; Pancreatitis; Myocardial Infarction; Histopathology.

\section{*Corresponding Author:}

Dr. Charan Kishor Shetty MBBS, MD,

Medical Lecturer, Department of Forensic Medicine, Faculty of Medicine, Universiti Sultan Zainal Abidin (Unisza), Kampus Kota, Kuala Terengganu, Terengganu, Malaysia 20400.

Tel: 609-6275546/609-6275740

Fax: 609-6275771/609-6275772

E-mail: shettykishor.k@rediff.com

Received: June 04, 2015

Accepted: June 15, 2015

Published: June 17, 2015

Citation: Shetty CK, Shetty SS (2015) Acute Haemorrhagic Pancreatitis Leading to Sudden Unexpected Death - A Case Report and Review of Literature. Int J Forensic Sci Pathol. 3(6), 139-141. doi: http://dx.doi. org/10.19070/2332-287X-1500033

Copyright: Shetty $\mathbf{C K}^{\circ}$ 2015. This is an open-access article distributed under the terms of the Creative Commons Attribution License, which permits unrestricted use, distribution and reproduction in any medium, provided the original author and source are credited.

\section{Introduction}

Sudden unexpected deaths form the subject of medicolegal investigation if they occur in apparently healthy individuals, wherein an autopsy would shed light regarding the cause of death. Sudden death has numerous causes most common system involved is Cardio-Vascular System [1]. Myocardial infarction tops the list of all cardiovascular pathologies not only in elderly, even young person's [1].

Acute pancreatitis is a condition peculiar to the pancreas in which there is destruction of the gland by enzymes escaping into its substance. It is characterised pathologically by haemorrhagic necrosis and inflammation of the pancreatic tissue and clinically by abdominal pain, vomiting and shock, which may cause death. The clinical diagnosis is usually based on the history, examination and investigations including serum and urinary amylase and ultrasonic and CT scans [2].

True sudden and unexpected death due to acute pancreatitis is rare. However most of the cases have acute hemorrhagic pancreatitis which is not problematic to diagnose during the autopsy. There are also anecdotal cases of fatty necroses in lipomatous heart as a cause of sudden death in acute pancreatitis [3]. However it seems that underlying hearth/vascular/brain disease is an important prerequisite for sudden death in acute pancreatitis [4].

We report a case of a 30 years male, apparently healthy who was found dead in his room. He was declared brought dead to the Kasturba medical college hospital, Manipal. Medico legal autopsy was done to know the cause of death.

\section{Case Report}

As-per the information furnished by police a 45 years old young male known alcoholic was found dead in his room which was locked from inside. An empty alcohol bottle was found near the dead body. He was brought dead to the Kasturba medical college hospital, Manipal which necessitated a medico legal autopsy.

On post mortem examination externally he was moderately built and nourished, conjunctiva was pale, lividity was present but was not fixed. Internally esophagus and oral cavity contained reddish brown fluid. Mucosa was congested with small erosions present over the lower one third of the esophagus. Stomach weighed 180 gram sand contained reddish brown fluid with absence of any abnormal odor; hemorrhagic ulcers (fresh) were present at several places over the stomach mucosa. Multiple atheromatous 
streaks and plaques were present over the mucosa of aorta. Heart weighed 290 grams. Valves were intact. Right ventricular thickness was $0.6 \mathrm{cms}$, and left ventricular thickness $2.0 \mathrm{cms}$. Right Coronary Artery showed 50\% block, $2 \mathrm{cms}$ from origin. Left anterior descending artery branch of the left coronary artery showed near total occlusion, $1.5 \mathrm{cms}$ from origin. White patch measuring $2 \mathrm{x}$ 1.7 was present over the anterior-lateral surface of the heart, 4 cms above apex. Pancreas weighed $95 \mathrm{Gms}$ and was hemorrhagic on cut section. Liver was enlarged with presence of yellowish discoloration on cut section.

Histopathological examination of the heart revealed features of acute myocardial infarction showing neutrophilic infiltrate along with areas of necrosis, diffuse interstitial edema and pale myocytes with fading nuclei and decreased striations as shown in (Figure. 1).

Histopathology of pancreatic tissue revealed patchy necrosis with hemorrhage and inflammatory cells as shown in (Figure. 2). Histopathological examination of the liver was suggestive of cirrhosis. Chemical analysis of routine viscera and body fluids was positive for alcohol. Blood was sent for biochemical examination of cardiac markers and for estimation of serum amylase levels both of which showed elevated levels results of which are given in (Table. 1)

Cause of death was given as myocardial infarction secondary to hemorrhagic pancreatic associated with alcohol intoxication.

\section{Discussion}

Many prior studies have suggested that the majority of deaths in severe acute haemorrhagic pancreatitis occur in the late phase of the disease as a result of pancreatic sepsis [2]. Conversely in the present case, the affected individual died during the very early phase of the disease with features suggestive of acute myocardial infarction.

Acute pancreatitis represents a spectrum of disease, ranging from a mild, transitory illness to a severe, rapidly progressive hemorrhagic form, with massive necrosis and mortality rates of up to $24 \%$. The reported incidence of acute pancreatitis diagnosed first at clinicopathologic autopsy ranges between $30 \%$ and $42 \%[3,4]$.

Acute pancreatitis (AP) in a subset of patients presents as sudden unexpected death and is diagnosed for the first time during autopsy [3]. The majority of cases of sudden unexpected death can usually be attributed to a past history of illness, clinical features or characteristic findings on postmortem examination. The forensic pathologist or the medical examiner faces the daunting task of determining the cause of death in those cases without any antecedent causes, suspected violence, suicide or homicide and with no clinical history of illness [5]. Systemic complications of acute pancreatitis involve pulmonary, cardiovascular and nervous system. Vagal reflexes commonly associated with acute pancreatitis

Figure 1. Histopathological examination of the heart showed features of acute myocardial infarction (100 x magnification).

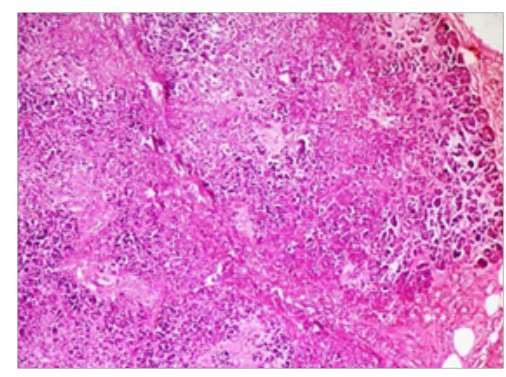

Figure 2. Histopathological examination of the pancreas revealed features suggestive of acute haemorrhagic pacreatitis (200 x magnification).

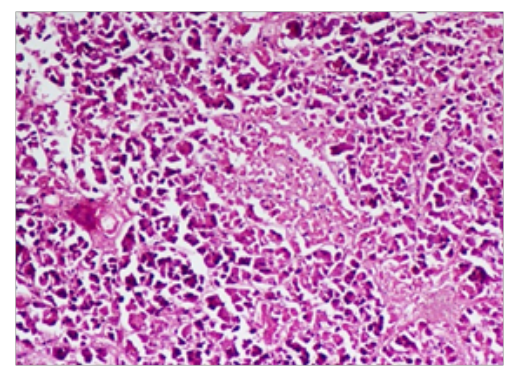

Table 1. Post mortem biochemical examination of blood for cardiac markers and serum amylase levels.

\begin{tabular}{|c|c|c|}
\hline Cardiac Markers & Detected Values & Normal Values \\
\hline CK $(\mathrm{MB}):$ & $283.8 \mathrm{ng} / \mathrm{ml}$ & $0.6-6.3 \mathrm{ng} / \mathrm{ml}$ \\
\hline Troponin-T & $1.88 \mathrm{ng} / \mathrm{ml}$ & $0.00-0.03 \mathrm{ng} / \mathrm{ml}$ \\
\hline NT PRO BNP & $183.8 \mathrm{pg} \cdot / \mathrm{ml}$ & $40-125 \mathrm{pg} / \mathrm{ml}$ \\
\hline Serum amylase & $979.0 \mathrm{U} / \mathrm{L}$ & $28-100 \mathrm{U} / \mathrm{L}$ \\
\hline
\end{tabular}


can cause cardiac damage by acting directly on the myocardium, indirectly altering coronary blood flow, or through the increased secretion of pancreatic proteolytic enzymes. Electrolytic abnormalities such as hypokalemia, hypocalcemia and hyponatremia are common in acute pancreatitis and can modify the repolarization phase on the ECG, but ST elevation in such cases is rarely seen [5].

Acute pancreatitis complicated with true myocardial infarction is very rare. In 2005, Korantzo Poulos et al., reported a case which was claimed to be the third case of MI with pancreatitis. In 2010 Sheng-Hsiung Sheu reported a similar case where coronary angiography and elective PTCA was done after the recovery from pancreatitis [6].

Pancreatic proteolytic enzymes including trypsin may directly damage the membrane of the myocyte with subsequent changes of cell permeability and possible cellular necrosis, as well as secondary electrical disturbance. These enzymes may change platelet adhesiveness and influence the coagulation system, thus leading to coronary thrombosis. Some studies revealed higher frequency of cardiovascular lesions in individuals with acute or chronic pancreatitis without relation to the common cardiovascular risk factors. There is also the possibility of exacerbation of these lesions during the pancreatic event. Although angiographic or morphologic analysis of patients with ST elevation in pancreatitis revealed normal coronary arteries in a high percentage of cases,a cardiovascular investigation is recommended in such cases [4-6].

Forensic pathologists sometimes face the problem of differentiating between (i) acute pancreatitis as a primary cause of death; (ii) secondary acute pancreatitis due to, for example, hypotension following cardiac surgery bypass or a massive myocardial infarction with acute heart failure; (iii) 'normal' post-mortem pancreatic autolysis which worsens in severity as times elapses after death [5]. The first two will look very similar obviously, both within the abdomen and also systemic complications (ARDS can compicate both acute pancreatitis and any other cause of prolonged hypotension), but in post-mortem autolysis necrosis will usually be restricted to the pancreas (and gut mucosa) without the more widespread fat necrosis throughout the abdomen, sometimes even more remotely, that accompanies true acute pancreatitis [7].

Histology would only help to differentiate pancreatic necrosis from peripancreatic necrosis which can make the exterior surface of the gland look necrotic, until the gland is sectioned, but the macroscopic appearances around the pancreas should usually suf- fice. Clearly it is important that clinicians pass on any relevant information on history, examination and investigation finding to the pathologist, and ideally a clinician should be present at the autopsy [8]. Sudden "apoplectic' death from acute myocardial infarction secondary to acute haemorrhagic pancreatitis is rare but is described in the literature and can occur in young patients [2, 3]. There is usually no true haemorrhage in acute pancreatitis, the haemorrhagic appearancs are due to digestion of haemoglobin producing methaemalbumen. The same process occurs in body wall bruising such as the Grey-Turner's and Cullen's signs around the flanks and umbilicus [6]. Good communication between clinicians and the pathologist is essential when trying to ascertain the true cause of death.

\section{Conclusion}

Determination of cause of death in natural deaths, particularly when the death occurred suddenly, unexpectedly, is an important part of forensic autopsy. Acute pancreatic disease is one of the such causes were careful attention should be paid to diagnose the particular disease to opine the cause of death. The above case report shows that although there are features suggestive of myocardial infarction it has to kept in mind that it was secondary to the acute hemorrhagic pacreatitis. This helps us in documenting a clear cause of death and rule out any foul play when encountered with such sudden unexpected death cases.

\section{References}

[1]. Heatley MK, Crane J (1989) Acute pancreatitis as a cause of sudden or unexpected death in Northern Ireland. Ulster Med J 58(1): 51- 55.

[2]. Roncati L, Gualandri G, Fortuni G, Barbolini G (2012) Sudden death and lipomatous infiltration of the heart involved by fat necrosis resulting from acute pancreatitis. Forensic Sci Int 217(1-3): e19-22.

[3]. Tsokos M, Braun C (2007) Acute pancreatitis presenting as sudden, unexpected death: an autopsy-based study of 27 cases. Am J Forensic Med Pathol 28(3): 267-270.

[4]. Rastogi P, Palimar V (2009) Sudden death due to acute haemorrhagic pancreatitis. J Indian Acad Forensic Med 31(4): 377-378.

[5]. Tümer AR, Dener C (2007) Diagnostic dilemma of sudden deaths due to acute hemorrhagic pancreatitis. J Forensic Sci 52(1): 180-182.

[6]. Kumar AV, Reddy GM, Kumar AA (2013) Acute pancreatitis complicated by acute myocardial infarction - A rare association. Indian Heart J 65(4): 474-477.

[7]. Shetty BS, Boloor A, Menezes RG, Shetty M, Menon A, et al. (2010) Postmortem diagnosis of acute haemorrhagic pancreatitis. J Forensic Leg Med 17(6): 316-320.

[8]. Murty OP, Agarwal A, Krishnan R (2008) Sudden death due to acute pancreatitis. Autopsy observations. Journal of Forensic Medicine and Toxicology 25(2): 47- 53 